\title{
Symmetry-dependent screening of surface plasmons in ultrathin supported films: The case of $\mathrm{Al} / \mathrm{Si}(111)$
}

\author{
Zhe Yuan \\ Department of Applied Physics, Chalmers University of Technology, SE-412 96, Göteborg, Sweden \\ Ying Jiang \\ International Center for Quantum Materials (ICQM), Peking University, 100871, Beijing, China \\ Yi Gao \\ Institute of Physics, Chinese Academy of Sciences, 100190, Beijing, China \\ Mikael Käll \\ Department of Applied Physics, Chalmers Tekniska Högskolan, SE-412 96, Göteborg, Sweden \\ Shiwu Gao* \\ Department of Physics, University of Gothenburg, SE-412 96, Göteborg, Sweden \\ (Received 28 October 2010; revised manuscript received 10 March 2011; published 29 April 2011)
}

\begin{abstract}
A joint theoretical and experimental study of plasmon excitations for $\mathrm{Al}$ overlayers on $\mathrm{Si}(111)$ has been carried out. The presence of the substrate is found to drastically modify the hybridization and charge density response of the surface plasmons of the metal overlayers. The symmetric mode, which is polarized toward the $\mathrm{Al} / \mathrm{Si}$ interface, is strongly damped in intensity and significantly redshifted in energy. However, the antisymmetric mode, which is polarized to the metal-vacuum interface, is essentially unaffected by the presence of the substrate. A low-energy acoustic plasmon mode is also found in a one monolayer $\mathrm{Al} \mathrm{film}$ and is almost unaffected by the substrate. The calculated plasmon dispersions with substrate are in good agreement with experimental data measured by electron energy loss spectroscopy. Our results suggest that interaction and screening at the subnanometer scale are symmetry dependent, a conclusion that may have general implications in other thin films and related structures.
\end{abstract}

DOI: $10.1103 /$ PhysRevB.83.165452

PACS number(s): 73.20.Mf, 71.45.Gm

\section{INTRODUCTION}

Surface plasmons of metallic thin films ${ }^{1-12}$ have been studied intensively due to their distinct and tunable properties. The latter feature is common to many other tunable plasmonic structures at nanometer scales. It is generally known that quantum-well states (QWSs) of thin films can modulate their single-particle spectra. Consequently, they also affect the collective excitations of the metal films. The energy dispersions of thin film plasmons have been studied in the literature by classical models. ${ }^{13-15}$ Recently, quantum-mechanical calculations based on linear response and the time-dependent local density approximation ${ }^{16-23}$ (LR-TDLDA) have also been performed for thin films as a function of thickness. ${ }^{11,12}$ The calculated energy dispersions are in good agreement with the classical expressions at large thickness. However when the thickness goes down to a few nanometers, the energy dispersions given by the quantum calculations start to deviate from the classical values and show quantum oscillations as a function of thickness. For ultrathin films with only a few atomic layers, a transition from three-dimensional surface plasmons to electron-hole pair excitations has been observed. These conclusions revealed the quantum-mechanical behavior of surface plasmons in ultrathin films in flat two-dimensional (2D) geometry. They may also have implications for surface plasmons in other types of shell-like structure in reduced dimensions.

Samples of metal films are usually grown and measured on substrates. ${ }^{1-9}$ Contact with a dielectric environment can modify the band structure, charge transfer, and electronic excitations of the thin metal films. These issues have been intensively investigated in the adsorption of alkali metal overlayers on metals $^{16-22}$ and semiconductor substrates. ${ }^{24,25}$ Electron energy loss spectroscopy (EELS) of silver and aluminum films on various substrates ${ }^{1-9}$ has indicated that the energies and linewidths of surface plasmons depend sensitively on the type of substrate on which the films are supported, and the film thicknesses. Very recently, high-quality films of a few Al monolayers (ML) have been grown on a Si(111) surface, 3,4 where their thickness can be controlled precisely down to $1 \mathrm{ML}$. Such a monolayer film offers a prototype system, in terms of both atomic and electronic structures, for a quasitwo-dimensional electron gas (2DEG). ${ }^{26-28}$ It also provides a model interface system where rigorous quantum-mechanical investigations can be carried out and detailed comparison with experiments can possibly be achieved.

So far, fully quantum-mechanical calculations for surface plasmons at interfaces have not been available due to two major obstacles: (1) the complicated mechanisms and unknown atomic structures involved in the growth process, ${ }^{29}$ and (2) the computational challenge in a quantum-mechanical description of large supercells of interface structures. ${ }^{30}$ For the case of $\mathrm{Al} / \mathrm{Si}(111)$, for example, deposition of $\mathrm{Al}$ on $\mathrm{Si}(111)$ lifts the $(7 \times 7)$ reconstruction of the substrate, yielding a commensurate structure with $(4 \times 4) \mathrm{Al}$ atoms in a $(3 \times$ 3) unitcell of the substrate. A fully quantum-mechanical treatment of this supercell is far beyond the reach of the 
current LR-TDLDA calculations. It is thus desirable to develop approximate schemes to treat such interface systems in order to gain deeper understanding of the plasmon excitations of the supported films.

In this paper, we extend our previous LR-TDLDA calculations for free-standing films to a jellium slab supported on a dielectric substrate to model Al films on the Si surface, which were measured by recent experiments. The ground-state electronic structure of the thin film is solved within density functional theory, while the Si substrate is simulated via the dielectric function of the substrate. Our description is similar to the recent approaches for nanoparticles and nanoshells ${ }^{31,32}$ with dielectric cores. Application to $\mathrm{Al} / \mathrm{Si}(111)$ reveals several effects of the substrate, including hybridization and charge transfer, and a symmetry- and frequency-dependent screening in the plasmon excitations. In particular, the symmetric plasmon mode, which is polarized to the $\mathrm{Al} / \mathrm{Si}$ interface, is strongly damped in intensity and redshifted in energy. In contrast, the antisymmetric mode, which is polarized toward the metal-vacuum interface, is much unaffected. Our results demonstrate that the screening effect on the atomic scale is energy and symmetry dependent. Similar symmetrydependent screening has also been found for the collective resonances in one-dimensional atomic chains. ${ }^{33}$

The rest of the paper is organized as follows. The experimental measurements are presented in Sec. II. The theoretical methods are outlined in Sec. III. This is followed by the results and comparison with experiments. In Sec. V, we give a brief summary. Details about the evaluation of the ground-state electronic structure and interactions in the presence of dielectrics are given in the Appendixes.

\section{EXPERIMENTS}

The experiments were carried out in a combined variabletemperature scanning tunneling microscope (VT-STM) (Omicron) and high-resolution EELS (HREELS) (LK-5000) ultrahigh-vacuum system $\left(p<1 \times 10^{-10} \mathrm{mbar}\right)$. The monolayer $\mathrm{Al}(111)-1 \times 1$ film was epitaxially grown on a $\mathrm{Si}(111)$ $1 \times 1$-terminated surface using the method described in Ref. 3 . After growth, the quality of the sample was checked by STM and low-energy electron diffraction (LEED). The HREELS measurement was then performed at room temperature (RT). The incident electron beam energies adopted in the HREELS measurement are 20 and $50 \mathrm{eV}$, with incident angles of $60^{\circ}$ and $55^{\circ}$ with respect to the direction normal to the surface, respectively. The energy dispersion of surface plasmons was obtained by rotating the analyzer, while fixing the incident angle. To enhance the signal-to-noise ratio, the energy resolution of the HREELS was further degraded to about $15 \mathrm{meV}$, judged from the full width at half maximum (FWHM) of the zero-loss peak. All the data shown in the work were obtained from a single sample.

The EEL spectra of the monolayer Al film exhibit two energy loss peaks, located below $1 \mathrm{eV}$ and around $11 \mathrm{eV}$ as shown in Figs. 1(a) and 1(b), respectively. The momentum transfer parallel to the surface, $q$, is calculated from the energies and angles of the incident and scattered electrons. The direction of $q$ is along $\Gamma M$ in the $\operatorname{Al}(111)$ surface Brillouin zone, as measured by LEED (inset in Fig. 1). For the
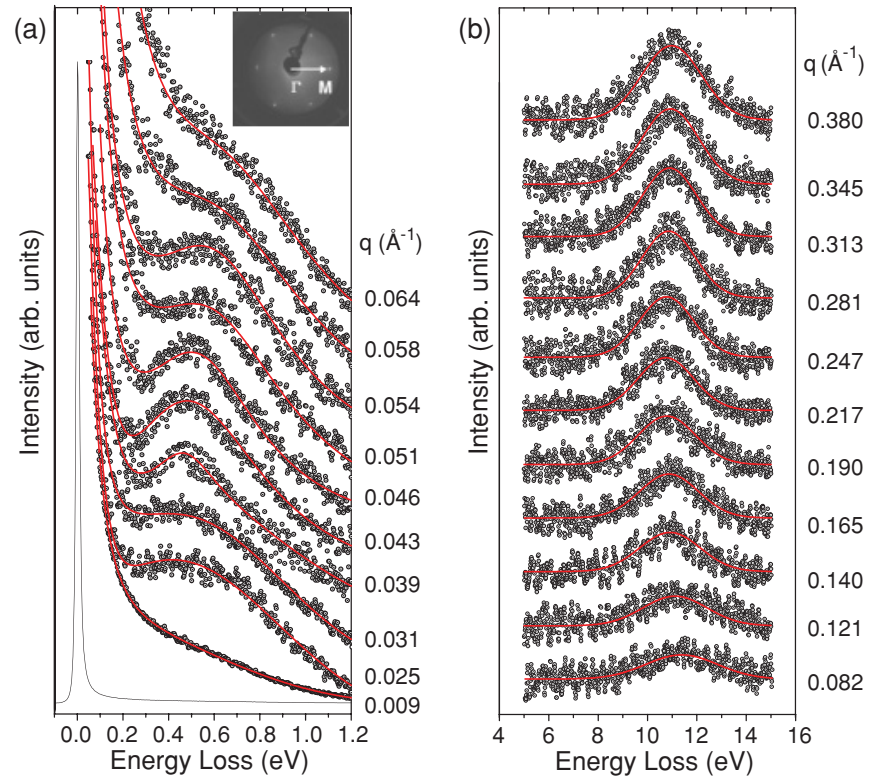

FIG. 1. (Color online) EEL spectra of the monolayer Al on $\mathrm{Si}(111)$ measured at RT at different scattering angles. (a) Incident angle, $60^{\circ}$; electron primary energy, $20 \mathrm{eV}$. Each spectrum is fitted by a Lorentzian peak (elastic peak) and a Gaussian peak (loss peak). Inset: LEED pattern obtained on the monolayer Al film, showing very sharp diffraction spots. The white arrow in the inset indicates the incident direction of the electron beam. (b) Incident angle, $55^{\circ}$; electron primary energy, $50 \mathrm{eV}$. Each spectrum is fitted by a single Gaussian peak. The momentum transfer parallel to the surface $q$ is calculated from the energies and angles of the incident and scattered electrons.

low-energy mode, the loss peak is superimposed on a Drude tail, resulting from the metallicity of the $\mathrm{Al}$ film. The peak position can be determined by fitting the EEL spectrum with a Lorentzian peak and a Gaussian peak. The low-energy mode disperses monotonically to higher energy with increasing $q$. On the other hand, the high-energy mode can be fitted by a single Gaussian peak with FWHM about $3 \mathrm{eV}$. This mode has a negative slope at small $q$ and positive slope at large $q$, which is very similar to the behavior of the surface plasmon of bulk $\mathrm{Al}(111)$. The corresponding energy dispersion curves of both modes are plotted in Fig. 5.

\section{THEORY}

Our theoretical model consists of a jellium slab with thickness $D$ and a dielectric substrate as schematically shown in the upper panel of Fig. 2. The LR-TDLDA method used in our previous publication for free-standing films ${ }^{11}$ has been modified to include the effect of the substrate. Our strategy is to decouple the quantum-mechanical response of the metal electrons from the classical electrodynamical response of the combined metal-dielectric systems. This is done in practice by using the classical response to an external potential as a background electrostatic potential, which acts as external perturbation to the electronic system of the supported films. In such a way, the LR-TDLDA calculation remains formally the same as in the free-standing film calculation, but the evaluation of the response functions has to be modified. Such 


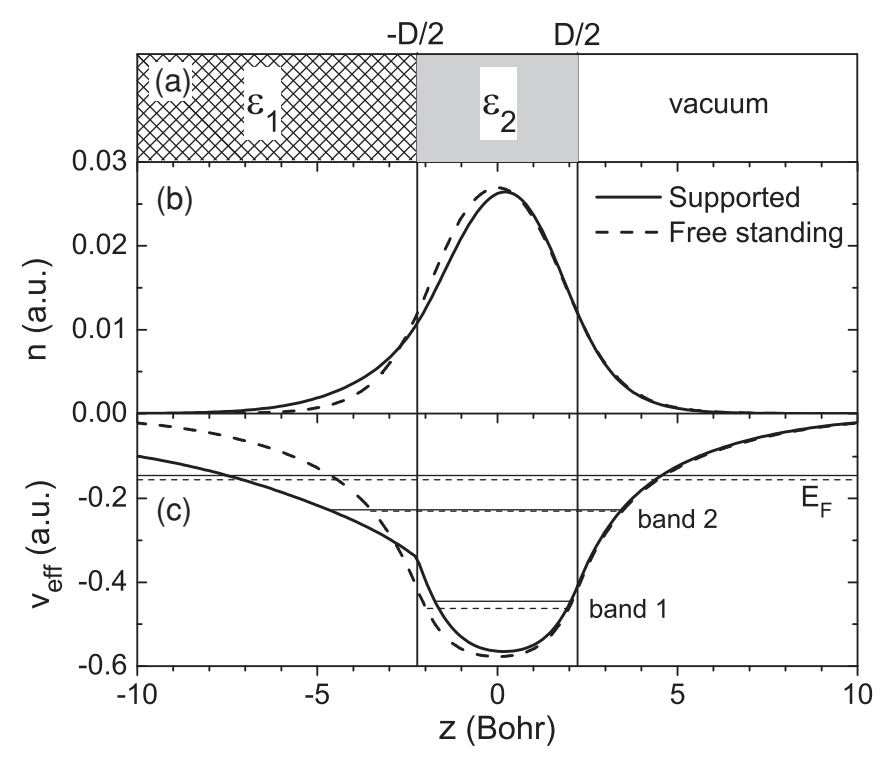

FIG. 2. Schematic structure (a), self-consistent electron density (b), and effective potential (c) of a free-standing (dashed lines) and supported (solid lines) Al monolayer on a Si(111) substrate. The static screening of Si with $\varepsilon_{S i}=11.65$ leads to charge transfer to the substrate. The thin solid (dashed) lines in (c) indicate the energy levels of the supported (free-standing) Al monolayer.

a separation between the classical and quantum-mechanical responses is possible due to the superposition principle of the linear electric field and the shared translational symmetry of the combined systems. Below we outline the basic steps of our theoretical method. Other details concerning the groundstate electronic structure and the evaluation of the Coulomb interaction kernel for the supported slab are given in the Appendixes.

\section{A. Electrodynamical response of the metal-dielectric system}

The electrodynamical response of the metal-dielectric interface can be described by classical electrodynamics. ${ }^{13,34,35}$ For 2D systems with translational symmetry, an arbitrary external perturbation can be expanded by Fourier transforms:

$$
\phi_{\mathrm{ext}}^{0}(\mathbf{r}, t)=\frac{1}{(2 \pi)^{2}} \int d^{2} q \int d \omega e^{i\left(\mathbf{q} \cdot \mathbf{r}_{\|}-\omega t\right)} \phi_{\mathrm{ext}}^{0}(z, q, \omega) .
$$

Here $\omega$ is the excitation energy and $q=|\mathbf{q}|$ is the wave vector parallel to the surface. Without losing generality, we consider the response to a perturbation in the form of $\phi_{\text {ext }}^{0}(z, q, \omega)=-2 \pi e^{q z} / q$, which may represent any sourceless perturbation in the surface region. ${ }^{36,37}$ This external potential induces polarizations in the dielectric substrate and the metal film, which can be described by the dielectric functions $\varepsilon_{1}(\omega)$ and $\varepsilon_{2}(\omega)$, respectively.

In the quasistatic approximation, the induced potential $\phi_{\text {ind }}^{\varepsilon}$ can be obtained by electrostatics. The total electrostatic potential $\phi_{\text {est }}=\phi_{\text {ext }}^{0}+\phi_{\text {ind }}^{\varepsilon}$ obeys the Laplace equation for each Fourier component,

$$
\frac{d}{d z}\left[\varepsilon(z, \omega) \frac{d}{d z} \phi_{\text {est }}(z, q, \omega)\right]+q^{2} \phi_{\text {est }}(z, q, \omega)=0 .
$$

The general solution of Eq. (2) can be formally written as

$$
\phi_{\text {est }}(z, q, \omega)=\left\{\begin{array}{lc}
\mathcal{A} e^{-q z}+\mathcal{B} e^{q z}, & z<-\frac{D}{2}, \\
\mathcal{C} e^{-q z}+\mathcal{D} e^{q z}, & -\frac{D}{2}<z<\frac{D}{2}, \\
\mathcal{E} e^{-q z}+\mathcal{F} e^{q z}, & z>\frac{D}{2} .
\end{array}\right.
$$

At $|z| \gg D / 2$ the induced potential $\phi_{\text {ind }}^{\varepsilon}(z, q, \omega)$ should vanish, so $\phi_{\text {est }}(z, q, \omega)$ approaches $\phi_{\text {ext }}^{0}(z, q, \omega)$, which makes $\mathcal{A}=$ 0 and $\mathcal{F}=-2 \pi / q$. The other four coefficients can be determined from the boundary conditions for the electrostatic potentials $\phi_{\text {est }}(z, q, \omega)$ and the electric displacements $\varepsilon(z, \omega) \frac{d}{d z} \phi_{\mathrm{est}}(z, q, \omega)$ at the two interfaces $z= \pm D / 2$. The total electrostatic potential of the combined system thus reads

$$
\phi_{\text {est }}(z, q, \omega)=\left\{\begin{array}{lc}
-\frac{2 \varepsilon_{2}}{\varepsilon_{2}+1} \frac{2 \gamma}{\varepsilon_{1}+\varepsilon_{2}} \frac{2 \pi}{q} e^{q z}, & z<-\frac{D}{2}, \\
-\frac{2 \gamma}{\varepsilon_{2}+1} \frac{2 \pi}{q}\left[e^{q z}+\frac{\varepsilon_{2}-\varepsilon_{1}}{\varepsilon_{2}+\varepsilon_{1}} e^{-q(z+D)}\right], & -\frac{D}{2}<z<\frac{D}{2}, \\
-\frac{2 \pi}{q}\left[e^{q z}+\gamma \frac{1-\varepsilon_{2}}{1+\varepsilon_{2}} e^{-q(z-D)}+\gamma \frac{\varepsilon_{2}-\varepsilon_{1}}{\varepsilon_{2}+\varepsilon_{1}} e^{-q(z+D)}\right], & z>\frac{D}{2},
\end{array}\right.
$$

with the common factor $\gamma$ defined by

$$
\gamma=\left(1+\frac{\varepsilon_{1}-\varepsilon_{2}}{\varepsilon_{1}+\varepsilon_{2}} \frac{\varepsilon_{2}-1}{\varepsilon_{2}+1} e^{-2 q D}\right)^{-1} .
$$

It is obvious that $\gamma=1$ when $\varepsilon_{2}=1$, which recovers the well-known case for a single dielectric medium.

The surface response function for the dielectric-metal interface is given by ${ }^{35}$

$$
g_{\varepsilon}(q, \omega)=\frac{\varepsilon_{\mathrm{eff}}(q, \omega)-1}{\varepsilon_{\mathrm{eff}}(q, \omega)+1},
$$

where the effective dielectric function $\varepsilon_{\text {eff }}$ of the combined system is defined as ${ }^{13}$

$$
\varepsilon_{\mathrm{eff}}(q, \omega)=\varepsilon_{2} \frac{\varepsilon_{1}+\varepsilon_{2}+\left(\varepsilon_{1}-\varepsilon_{2}\right) e^{-2 q D}}{\varepsilon_{1}+\varepsilon_{2}-\left(\varepsilon_{1}-\varepsilon_{2}\right) e^{-2 q D}} .
$$

The imaginary part of the surface response function corresponds to the energy absorption of the classical system. After some algebra, we obtain

$$
\operatorname{Im} g_{\varepsilon}(q, \omega)=\operatorname{Im}\left[\gamma\left(\frac{\varepsilon_{1}-\varepsilon_{2}}{\varepsilon_{1}+\varepsilon_{2}} e^{-2 q D}+\frac{\varepsilon_{2}-1}{\varepsilon_{2}+1}\right)\right] .
$$




\section{B. Quantum-mechanical response of the electrons in the supported slab}

The simplest way to treat the quantum-mechanical response of the electronic subsystem is to replace the local dielectric function of the metal slab, $\varepsilon_{2}$, by the quantum-mechanical description of their electrons. The details about the calculation of the ground-state electronic structure can be found in Appendix A. The linear response of the metal slab is carried out using the electrostatic potential of the dielectric background as the external perturbation, $\phi_{\text {ext }}=\phi_{\text {est }}$. In the following formulation, we keep $\varepsilon_{2}$ to mimic possible core polarization beyond the free-electron approximation. ${ }^{34,37}$ For aluminum, we can simply set $\varepsilon_{2}=1$.

The quantum-mechanical response of the electronic subsystem is defined by the induced electron density $\delta n$ subjected to the electrostatic potential Eq. (4),

$$
\delta n(z, q, \omega)=\int d z^{\prime} \chi\left(z, z^{\prime}, q, \omega\right) \phi_{\mathrm{est}}\left(z^{\prime}, q, \omega\right) .
$$

The density response function $\chi\left(z, z^{\prime}, q, \omega\right)$ contains excitation properties of the many-electron systems.

As in the free-standing film, the density response $\delta n$ is also related to the variation of the effective potential of the supported film, $\phi_{\text {eff }}\left(z^{\prime}, q, \omega\right)$, via the noninteracting density response function $\chi_{0}\left(z, z^{\prime}, q, \omega\right)$,

$$
\begin{aligned}
\delta n(z, q, \omega)= & \int d z^{\prime} \chi_{0}\left(z, z^{\prime}, q, \omega\right) \phi_{\mathrm{eff}}\left(z^{\prime}, q, \omega\right) \\
= & \int d z^{\prime} \chi_{0}\left(z, z^{\prime}, q, \omega\right)\left[\phi_{\mathrm{est}}\left(z^{\prime}, q, \omega\right)\right. \\
& \left.+\delta \phi_{\mathrm{C}}\left(z^{\prime}, q, \omega\right)+\delta \phi_{\mathrm{xc}}\left(z^{\prime}, q, \omega\right)\right] .
\end{aligned}
$$

The second and third terms correspond to the changes of the Coulomb and exchange-correlation potentials induced by $\delta n$, respectively,

$$
\begin{aligned}
\delta \phi_{\mathrm{C}}(z, q, \omega) & =\int d z^{\prime} K_{\mathrm{C}}\left(z, z^{\prime}, q, \omega\right) \delta n\left(z^{\prime}, q, \omega\right), \\
\delta \phi_{\mathrm{xc}}(z, q, \omega) & =\int d z^{\prime} K_{\mathrm{xc}}\left(z, z^{\prime}, q, \omega\right) \delta n\left(z^{\prime}, q, \omega\right),
\end{aligned}
$$

where $K_{\mathrm{C}}$ and $K_{\mathrm{xc}}$ are the electron-electron interaction kernels. Within the TDLDA, the latter is given by

$$
K_{\mathrm{xc}}\left(z, z^{\prime}, q, \omega\right)=\left.\frac{\partial v_{\mathrm{xc}}}{\partial n}\right|_{n=n(z)} \delta\left(z-z^{\prime}\right) .
$$

With the dielectric substrate, the Coulomb kernel $K_{\mathrm{C}}\left(z_{1}, z_{2}, q, \omega\right)$ between two points is slightly more complicated due to the polarization of the dielectric, or the potential of the image charges. As such, $K_{\mathrm{C}}$ depends on the dielectric functions of all components and on the thickness of the thin film. The evaluation of the Coulomb kernel is given in detail in Appendix B. Combining Eqs. (9), (10), (11), and (12), we arrive at a Dyson equation,

$$
\begin{aligned}
\chi\left(z, z^{\prime}, q, \omega\right)= & \chi_{0}\left(z, z^{\prime}, q, \omega\right)+\iint d z_{1} d z_{2} \chi_{0}\left(z, z_{1}, q, \omega\right) \\
& \times K\left(z_{1}, z_{2}, q, \omega\right) \chi\left(z_{2}, z^{\prime}, q, \omega\right),
\end{aligned}
$$

with $K=K_{\mathrm{C}}+K_{\mathrm{xc}}$. The computation of $\chi_{0}$ from the groundstate band structure of the supported film and the solution of Eq. (14) are formally the same as in the free-standing systems. $^{11}$

\section{Electron energy loss spectrum and loss function}

In EELS experiments, the scattering cross section $\sigma$ per unit solid angle $d \Omega$ per energy $d \omega$ is given by ${ }^{38}$

$$
\begin{aligned}
\frac{d^{2} \sigma}{d \Omega d \omega}= & \frac{m^{2} e^{2} v_{\perp}^{2}}{2 \pi \cos \theta_{I}}\left(\frac{k_{S}}{k_{I}}\right) \\
& \times \frac{\left|v_{\perp} q\left(R_{I}+R_{S}\right)+i\left(R_{I}-R_{S}\right)\left(\omega-\mathbf{v}_{\|} \cdot \mathbf{q}\right)\right|^{2}}{\left[v_{\perp}^{2} q^{2}+\left(\omega-\mathbf{v}_{\|} \cdot \mathbf{q}\right)^{2}\right]^{2}} \frac{P(q, \omega)}{q^{2}},
\end{aligned}
$$

where $\theta_{I}$ is the incident angle, $\mathbf{v}_{\perp}$ and $\mathbf{v}_{\|}$are the normal and parallel velocities of the incoming electrons, $k_{I}$ and $k_{S}$ are the wave vector magnitudes of the incident and scattered electrons, and $\mathbf{q}$ is the parallel momentum transferred to the surface, $q=|\mathbf{q}|=\left|\mathbf{k}_{I}-\mathbf{k}_{S}\right| . \quad R_{I}$ and $R_{S}$ are the specular elastic scattering amplitudes after and before reflection, respectively. The prefactor in Eq. (15) depends only on the scattering geometry, while the function $P(q, \omega)$ is the probability of generating electronic excitations. It can be calculated from ${ }^{37}$

$$
\begin{aligned}
P(q, \omega)= & -\frac{q^{2}}{4 \pi^{2}} \operatorname{Im} \iint d z d z^{\prime} \phi_{\mathrm{eff}}^{*}(z, q, \omega) \\
& \times \chi_{0}\left(z, z^{\prime}, q, \omega\right) \phi_{\mathrm{eff}}\left(z^{\prime}, q, \omega\right) \\
\equiv & -\frac{q}{2 \pi} \operatorname{Im} g_{e}(q, \omega) .
\end{aligned}
$$

Here the surface loss function due to the metal electrons is defined by

$$
\begin{aligned}
\operatorname{Im} g_{e}(q, \omega)= & -\frac{q}{2 \pi} \operatorname{Im} \iint d z d z^{\prime} \phi_{\mathrm{eff}}^{*}(z, q, \omega) \\
& \times \chi_{0}\left(z, z^{\prime}, q, \omega\right) \phi_{\mathrm{eff}}\left(z^{\prime}, q, \omega\right) \\
= & -\frac{q}{2 \pi} \operatorname{Im} \int d z \phi_{\mathrm{eff}}^{*}(z, q, \omega) \delta n(z, q, \omega) .
\end{aligned}
$$

In the absence of dielectrics, the Coulomb and exchange correlation kernels are real symmetric, so that Eq. (17) can be simplified as

$$
\begin{aligned}
\operatorname{Im} g_{e}(q, \omega)= & -\frac{q}{2 \pi} \operatorname{Im} \iint d z d z^{\prime} \phi_{\mathrm{ext}}^{*}(z, q, \omega) \\
& \times \chi\left(z, z^{\prime}, q, \omega\right) \phi_{\mathrm{ext}}\left(z^{\prime}, q, \omega\right) .
\end{aligned}
$$

Unfortunately, this simplification is not possible in this work due to the complex Coulomb kernel. The more general definition in Eq. (17) has to be used to calculate the loss function.

The total energy loss spectrum consists of the quantummechanical contribution of jellium electrons, $\operatorname{Im} g_{e}$, and the absorption of the dielectric background $\operatorname{Im} g_{\varepsilon}$ in Eq. (8). Therefore the total surface loss function of the metal-dielectrics system reads

$$
\begin{aligned}
\operatorname{Im} g(q, \omega)= & -\frac{q}{2 \pi} \operatorname{Im} \int d z \phi_{\mathrm{eff}}^{*}(z, q, \omega) \delta n(z, q, \omega) \\
& +\operatorname{Im}\left[\gamma\left(\frac{\varepsilon_{1}-\varepsilon_{2}}{\varepsilon_{1}+\varepsilon_{2}} e^{-2 q D}+\frac{\varepsilon_{2}-1}{\varepsilon_{2}+1}\right)\right] .
\end{aligned}
$$


Note that all the above relations hold for frequency-dependent dielectric functions. For the special case with $\varepsilon_{1}=\varepsilon_{2}=1.0$, it reduces to the free-standing jellium slab we studied before. This limiting case is useful for checking the code implementation. The sum rule for the surface response function ${ }^{30}$ is no longer fulfilled with the dielectric background.

\section{COMPUTATIONAL RESULTS AND DISCUSSION}

First, we apply the jellium-dielectrics model to $1 \mathrm{ML}$ of $\mathrm{Al}$ film on $\mathrm{Si}(111){ }^{4}$ The complex dielectric function of silicon is taken from the optical measurement ${ }^{39}$ and its energy dependence is interpolated to arbitrary frequencies using the cubic spline method. The Al layer is simulated by a jellium slab with electron density $r_{s}=2.07$ and $D=4.5 \mathrm{bohr}$ for the $\mathrm{Al}(111)$ surface. The thicknesses of the substrate and the vacuum region are fixed at $41 \mathrm{bohr}$, which is converged for the simulations of these regions.

The lower part of Fig. 2 shows the electron density [Fig. 2(b)] and the effective potential [Fig. 2(c)] of the free-standing (dashed lines) and supported (solid lines) Al ML in the ground state. The Si substrate significantly reduces the electronic potential in the substrate due to the static screening of silicon $\left(\varepsilon_{\mathrm{Si}}=11.65\right)$. As a result, the total electron densities are spilled out toward the substrate, leading to charge transfer into the substrate. Such a charge transfer is also found to play a role in the surface plasmons ${ }^{5}$ of silver nanodisks. The derivative of the electron potential is discontinuous at the $\mathrm{Si}-\mathrm{Al}$ interface due to the boundary condition. In contrast, the electron density and effective potential of the Al-vacuum interface are unaffected by the presence of the substrate for such a thin film as $1 \mathrm{ML}$. A similar jellium-on-slab model was used before in the study of charge transfer and work function change of metal-semiconductor interfaces. ${ }^{24,25}$ The results in Fig. 2 are in agreement with previous density functional theory calculations with an atomic slab modeling the semiconductor substrate.

Figure 3 displays the surface loss functions $\operatorname{Im} g(q, \omega)$ at different $q$ values of the free-standing [Fig. 3(a)] and the supported Al monolayer [Fig. 3(b)]. Classically, thin films generally support two surface plasmon modes, the symmetric $\left(\omega_{-}\right)$ and antisymmetric $\left(\omega_{+}\right)$modes, $\omega_{ \pm}=\omega_{\mathrm{sp}} \sqrt{1 \pm \exp (-q D)}$, where $\omega_{\text {sp }}$ is the frequency of the surface plasmon. Both modes show up in our quantum calculations in the upper panel, but are split up due to the strong electronic interaction between the two surfaces. ${ }^{11,12}$ The symmetric mode exhibits a large dispersion from $3 \mathrm{eV}$ at $q=0.038 \AA^{-1}$ to $8 \mathrm{eV}$ at $q=0.302 \AA^{-1}$ and has high intensity at small $q$ due to the fact that decays into electron-hole pairs are forbidden in the low-energy regime of the 2DEG. The high-energy branch appears as a broad band at around $12 \mathrm{eV}$ and shows little dispersion. It splits into interband transitions at small $q$. In addition, a third mode is also discernible at 5-6 eV without much dispersion. Parity-resolved analysis shows that this mode consists of both the symmetric and antisymmetric components. It is an electron-hole pair type excitation. We should mention here that the symmetric mode is broadened dramatically at $q \geqslant 0.151 \AA^{-1}$, due to the onset of electron-hole pair excitations in this regime. Finally, there is a weak mode of linear energy dispersion from 0 to $2 \mathrm{eV}$, which can be associated with the acoustic plasmon

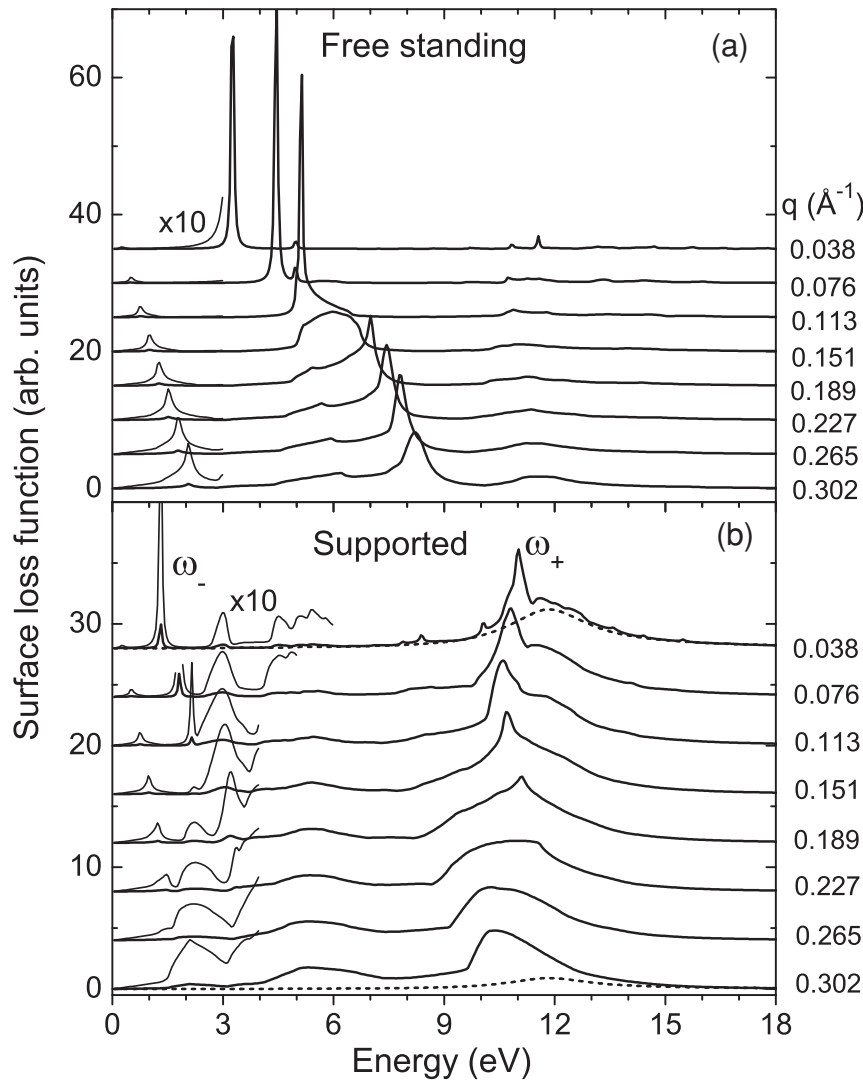

FIG. 3. Surface loss functions $\operatorname{Im} g(q, \omega)$ of the free-standing (a) and supported (b) Al monolayer as functions of the excitation energy $\omega$ and momentum transfer $q$. The dashed lines in (b) show the surface loss function of the bare $\mathrm{Si}$ substrate without the $\mathrm{Al}$ monolayer.

mode. Such a mode was theoretically predicted and has been measured recently on $\mathrm{Be}(0001)$ (Ref. 40) and $\mathrm{Au}(111) .{ }^{41}$ It can be associated with the collective density oscillations within 2D surface bands. ${ }^{42,43}$ In the case of an $\mathrm{Al}$ monolayer, this collective mode can be attributed to density oscillations within the two partially occupied bands as shown in Fig. 2(c). The linear dispersion coefficient of the acoustic mode, 0.47 a.u., is a little above the Fermi velocity of band $2\left(v_{F}=0.39\right.$ a.u.), but is consistent with the previous theory for noble metal surfaces. $^{43}$

For comparison, the loss functions of the supported film [Fig. 3(b)] exhibit quite distinct features from those of freestanding films. First of all, the antisymmetric mode becomes much more prominent in the spectra, and its energy is slightly redshifted. The large increase of intensity in the high-energy region results from the contributions from the energy loss in the silicon substrate, which is indicated by the dashed lines in the same panel. The substrate contribution is especially significant at small $q$, due to the deep penetration of the induced potential. At increasing $q$, it decreases rapidly due to the decay of the electron potential. In contrast, the symmetric mode is strongly damped in intensity and its energy is substantially redshifted by about $70 \%$, which is comparable to a scaling factor $1 / \sqrt{\varepsilon_{\mathrm{Si}}+1}$, as expected by the classical model. This mode disappears as it enters the absorption region of $\mathrm{Si}$ above $2 \mathrm{eV}$. The weak mode of electron-hole excitations at 5-6 eV 


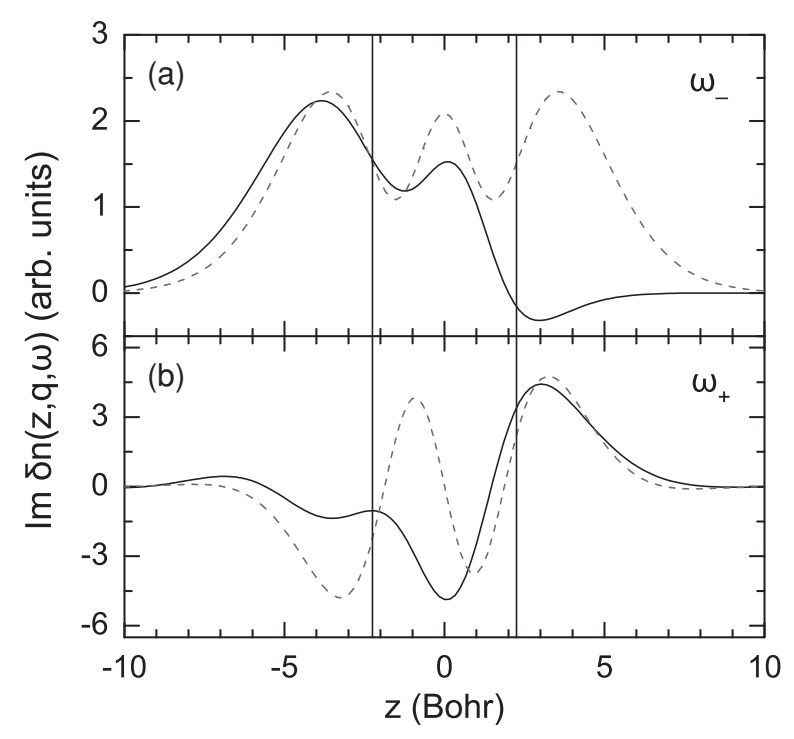

FIG. 4. Induced charge density of the symmetric $\omega_{-}$and antisymmetric $\omega_{+}$modes at $q=0.038 \AA^{-1}$ as marked in Fig. 3 for the free-standing (dashed lines) and supported (solid lines) Al monolayer.

is still visible, but is much more broadened due to coupling to the substrate. The acoustic plasmon peak becomes even weaker due to the substrate screening. Comparison between the isolated and supported films suggests that the effect of the substrate is very different for different modes. It strongly depends on the energy and symmetry of the electronic response of each mode. This conclusion is very different from what is usually expected and treated by classical models.

In order to gain insight into the mode-dependent screening of the substrate, the induced electron densities of the symmetric and antisymmetric mode at $q=0.038 \AA^{-1}$ are shown in Fig. 4. The induced density of the symmetric mode is clearly polarized at the $\mathrm{Si}-\mathrm{Al}$ interface, while that of the antisymmetric mode is pushed toward the vacuum region. This polarization can be understood from the plasmon hybridization model. ${ }^{11,44}$ The antisymmetric mode has thus roughly a frequency of $\omega_{+} \simeq \frac{\omega_{p}}{\sqrt{2}}$, the frequency of the surface plasmon, and is not much affected by the presence of the silicon substrate. In contrast, the frequency of the symmetric mode, which is localized toward the substrate-metal interface, is substantially redshifted as shown in Fig. 3. Charge polarization explains why the two modes experience different screening in the presence of the substrate. In addition, electron transfer to the substrate, which reduces the effective electron density of the $\mathrm{Al}$ monolayer, may also be responsible to the overall redshifts of energies in both modes.

Figure 5 summarizes the dispersions of the surface plasmons for the free-standing and supported Al ML together with comparison with experimental data and classical models. ${ }^{13}$ The energy dispersion of the antisymmetric mode at $11 \mathrm{eV}$, which is the main feature of the energy loss functions, is in excellent agreement with that measured by the experiment. This mode shows a minor negative dispersion, which is characteristic for the surface plasmons of free-electron-like metals. ${ }^{37}$ Inclusion of substrate screening is essential to reach better agreement with experiment for this mode, as the energies

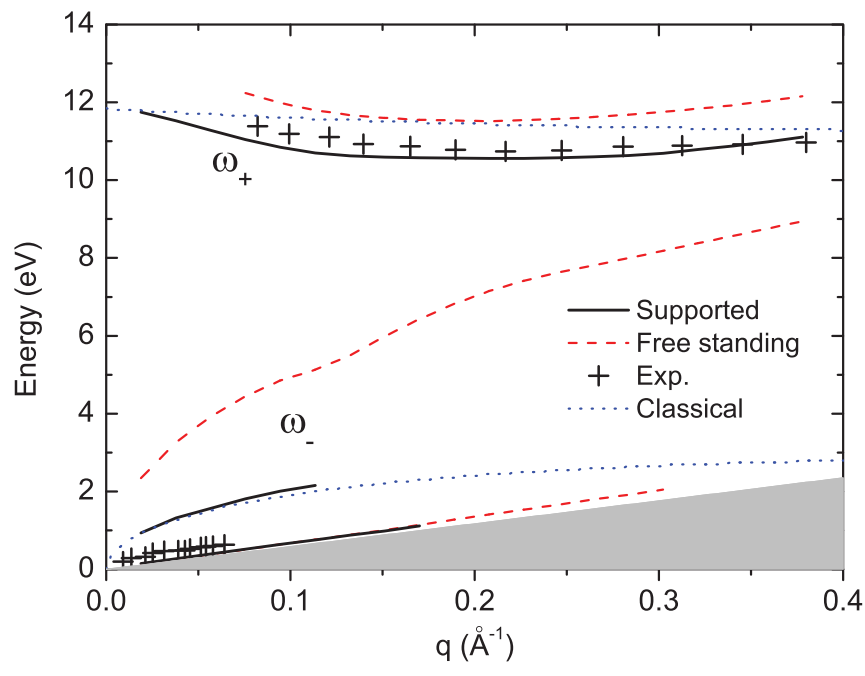

FIG. 5. (Color online) Energy dispersions of the surface plasmons for the free-standing (dashed lines) and supported (solid lines) Al monolayer. The experimental data $(+)$ are also plotted for comparison. The classical dispersions (dotted lines) are obtained by the Gadzuk model (Ref. 13) with the same dielectric function for $\mathrm{Si}$ and a Drude model for the Al monolayer, where $\varepsilon_{\mathrm{Al}}(\omega)=$ $1-\omega_{p}^{2} / \omega(\omega+i \eta)$ with $\omega_{p}=15.82 \mathrm{eV}$ and $\eta=27 \mathrm{meV}$. The gray area indicates the region of $(q, \omega)$ plane where electron-hole pairs can be created within the upper occupied state [band 2 in Fig. 2(c)] in the supported Al monolayer.

of the free-standing films are higher by about $1 \mathrm{eV}$. The symmetric mode between 1 and $2 \mathrm{eV}$, which is weak but visible in the theoretical calculations, is not measured in the loss spectra. This might indicate that our model does not fully account for all possible effects in the low-energy regime. Or perhaps the measurement in this regime is not sensitive enough, because the calculation indicates that the symmetric mode is indeed strongly damped. It is also known that the low-energy mode is very sensitive to interface roughness and in-plane scattering. ${ }^{45}$ It is interesting to note that the acoustic plasmon is almost unaffected by the presence of the substrate. In the experiment, a similar low-energy mode with linear dispersion has been measured and might be assigned to the acoustic plasmon mode. We should point out here that the low-energy excitation is sensitive to the atomic bonding and band structures of the interface, which are not fully included in the present model. It remains interesting in the future to study these low-energy modes using fully atomistic models together with LR-TDLDA calculations. ${ }^{30}$

To see the evolution of the surface plasmons with thickness in the presence of substrate, Fig. 6 shows the energy dispersions of the main surface mode $\omega_{+}$for $D=1,2$, and $3 \mathrm{ML}$ of $\mathrm{Al}(111)$ on the silicon surface. It can be seen that all three dispersions approach the same one at small $q$, due to the deep penetration of the probing electrons. In this limit, the loss function is completely dominated by the contribution from the silicon substrate. At intermediate $q=0.1-0.3 \AA^{-1}$, the plasmon energies increase with the thickness. This blueshift in energy reflects the change of free-electron densities in the overlayers and the gradual reduction of substrate effects. This trend for the supported films is opposite to the thickness dependence of the free-standing films, where a redshift was 


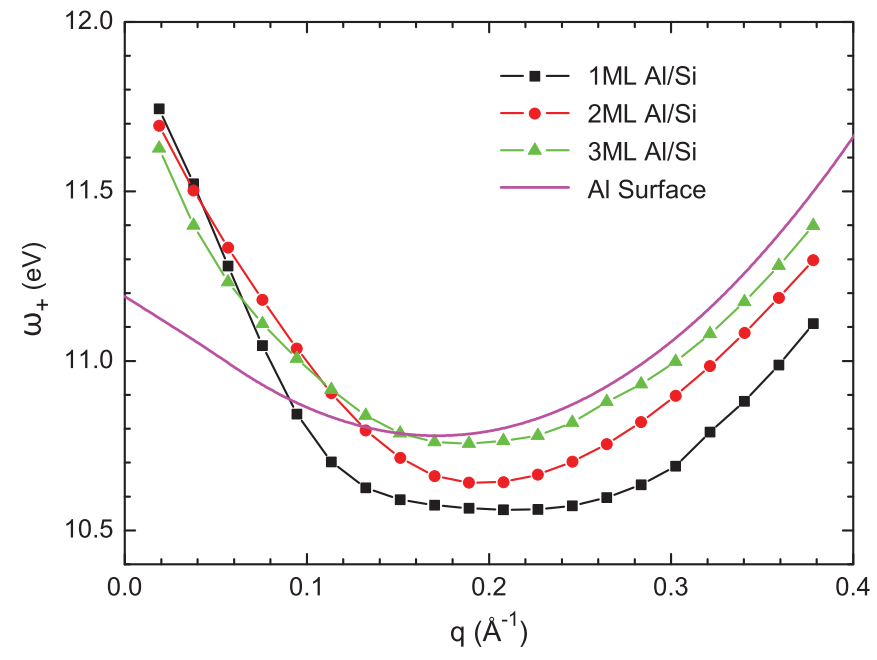

FIG. 6. (Color online) Plasmon dispersions for the high-energy mode of $\mathrm{Al} / \mathrm{Si}(111)$ with thickness $D=1,2$, and $3 \mathrm{ML}$. The solid line shows the plasmon dispersion of the $\mathrm{Al}(111)$ surface, modeled by a thick jellium slab with $D=43 \AA$.

generally found as $D$ increases. ${ }^{11}$ It is an indication of the electron transfer at the interface, which absorbs a fraction of free electrons into the substrate at $1 \mathrm{ML}$, reducing the effective electron density in Al. The charge transfer becomes less important in thicker films. In addition, as $D$ increases, the dynamical screening of the substrate also has less influence on the $\omega_{+}$mode, whose electron oscillations are localized mainly at the Al-vacuum interface. At $D \geqslant 3 \mathrm{ML}$, the dispersion of the Al film approaches the surface plasmon of the semi-infinite $\mathrm{Al}(111)$ surface, and the effect of the substrate gradually disappears. This limit occurs earlier in the large- $q$ regime, and is obvious for $q>0.2 \AA^{-1}$ at $3 \mathrm{ML}$. The evolution of energy dispersion in Fig. 6 indicates the effects of interface charge transfer and substrate screening on the surface plasmon excitations. Such effects may generally occur in other types of structure such as coated nanoparticles, nanoshells, and molecular-metal interfaces.

\section{SUMMARY}

We have performed a joint experimental and theoretical study of plasmon excitations of supported $\mathrm{Al}$ monolayer on a $\mathrm{Si}$ substrate. A low-energy mode below $1 \mathrm{eV}$ and a high-energy one around $11 \mathrm{eV}$ are found in the electron energy loss spectra. Within the LR-TDLDA, we have developed a jellium-dielectric model to treat the effect of substrate screening on the plasmon excitations. The calculated energy dispersion of the supported thin films is in good agreement with the measured data. The high- and low-energy modes in the measured spectra correspond to the antisymmetric mode and the acoustic surface plasmon mode, respectively. The substrate drastically modifies the electronic structure of the ground state and the dynamic response to the external field. In the ground state, the silicon substrate induces an electron transfer from the metal to the substrate due to static screening. The substrate also modifies the hybridization of surface plasmons. The symmetric mode, whose charge oscillation is mainly localized at the substrate-metal interface, is strongly damped by the substrate. In contrast, the antisymmetric mode is much less affected because its charge response is polarized toward the vacuum region. Increasing film thickness leads to charge transfer back to the film and blueshift of plasmon frequencies. The strong damping of the symmetric mode also explains the absence of the symmetric mode in the energy loss spectra. These conclusions may be general to surface plasmons at metal-semiconductor interfaces and in other types of structure.

\section{ACKNOWLEDGMENTS}

The authors would like to thank Kehui Wu for communications. This work has been supported by the Functional Meta Materials project, funded by the Foundation for the Strategic Research (SSF), the Institutional Grant Program of STINT of Sweden, and the 973 plasmonics program of China.

\section{APPENDIX A: GROUND-STATE ELECTRONIC STRUCTURE OF THE SUPPORTED FILMS}

In this appendix, we present the solution of the groundstate electronic structure of a supported jellium slab. The translational symmetry parallel to the surfaces allows us to solve the quantum-well states of the slab separately using the following 1D Kohn-Sham equation:

$$
\left[-\frac{1}{2} \frac{d^{2}}{d z^{2}}+v_{\text {eff }}(z)\right] \psi_{n}(z)=\epsilon_{n} \psi_{n}(z),
$$

where $\epsilon_{n}$ and $\psi_{n}$ are the one-electron eigenenergy and wave function, respectively. The effective electron potential $v_{\text {eff }}(z)$ consists of the electrostatic term $\varphi(z)$ and the exchangecorrelation term $v_{\mathrm{xc}}(z)$,

$$
v_{\text {eff }}(z)=\varphi(z)+v_{\text {xc }}(z)
$$

Here $v_{\mathrm{xc}}(z)$ is described within the local density approximation by Wigner as used before ${ }^{11}$ and it does not depend on the dielectric medium. The electrostatic potential $\varphi(z)$ is obtained by solving the Poisson equation,

$$
\frac{\partial}{\partial z}\left[\varepsilon(z) \frac{\partial}{\partial z} \varphi(z)\right]=-4 \pi\left[n(z)-n_{\text {ion }}(z)\right],
$$

where $n(z)$ and $n_{\text {ion }}(z)$ are the densities of electrons and positive ions, respectively. Equation (A3) implies continuation of the electric displacement field at each interface $z=z_{i}=$ $\pm D / 2$,

$$
\varepsilon\left(z_{i}^{-}\right) \varphi^{\prime}\left(z_{i}^{-}\right)=\varepsilon\left(z_{i}^{+}\right) \varphi^{\prime}\left(z_{i}^{+}\right) .
$$

The spatial dependence of the dielectric constant $\varepsilon(z)$ is defined by

$$
\varepsilon(z)=\left\{\begin{array}{lc}
\varepsilon_{1}, & z<-D / 2, \\
\varepsilon_{2}, & -D / 2<z<D / 2, \\
1, & z>D / 2 .
\end{array}\right.
$$

Here $\varepsilon_{1}$ is the static dielectric constant of the substrate. We also introduce $\varepsilon_{2}$ in the jellium slab region to mimic possible polarizations of the metal ion cores. The right-hand side of the film is vacuum $(\varepsilon=1)$; the impact electrons come from there in the EELS experiments. In this paper $\varepsilon_{2}=1.0$ for $\mathrm{Al}$ has been used, but it is kept as an arbitrary constant in our formulation. 


\section{APPENDIX B: EVALUATION OF THE COULOMB KERNEL FOR TDLDA CALCULATION}

Here we derive the Coulomb kernel in the presence of dielectrics. In general, the Coulomb kernel $K_{\mathrm{C}}\left(\mathbf{r}, \mathbf{r}^{\prime}\right)$ represents an effective electric field at $\mathbf{r}$, which is generated by a point charge at $\mathbf{r}^{\prime}$. It can be obtained by solving the following Poisson equation:

$$
\nabla \cdot\left[\varepsilon(\mathbf{r}, \omega) \nabla K_{\mathrm{C}}\left(\mathbf{r}, \mathbf{r}^{\prime}, \omega\right)\right]=-4 \pi \delta\left(\mathbf{r}-\mathbf{r}^{\prime}\right) .
$$

Due to the translational symmetry parallel to surfaces, $K_{\mathrm{C}}$ is expanded in terms of the transverse momentum $\mathbf{q}$,

$$
K_{\mathrm{C}}\left(\mathbf{r}, \mathbf{r}^{\prime}, \omega\right)=\frac{1}{4 \pi^{2}} \int d^{2} q e^{i \mathbf{q} \cdot\left(\mathbf{r}_{\|}-\mathbf{r}_{\|}^{\prime}\right)} K_{\mathrm{C}}\left(z, z^{\prime}, q, \omega\right),
$$

and the reduced one-dimensional Poisson equation can be solved piecewise (for $z \neq \pm D / 2$ ) with appropriate continuous boundary conditions at the interfaces,

$$
\frac{d^{2}}{d z^{2}} K_{\mathrm{C}}\left(z, z^{\prime}, q, \omega\right)-q^{2} K_{\mathrm{C}}\left(z, z^{\prime}, q, \omega\right)=-\frac{4 \pi}{\varepsilon(z, \omega)} \delta\left(z-z^{\prime}\right) \text {. }
$$

For $z^{\prime}<-D / 2$, the particular solution of Eq. (B3) $2 \pi /(\varepsilon q) \exp \left(-q\left|z-z^{\prime}\right|\right)$ exists only at $z<-D / 2$ and the whole solution can be formally written as

$$
\begin{aligned}
& K_{\mathrm{C}}\left(z, z^{\prime}, q, \omega\right) \\
& \quad= \begin{cases}\frac{2 \pi}{\varepsilon_{1} q} e^{-q\left|z-z^{\prime}\right|}+\mathcal{A} e^{q z}+\mathcal{A}^{\prime} e^{-q z}, & z<-\frac{D}{2}, \\
\mathcal{B} e^{q z}+\mathcal{C} e^{-q z}, & -\frac{D}{2}<z<\frac{D}{2}, \\
\mathcal{D} e^{-q z}+\mathcal{D}^{\prime} e^{q z}, & z>\frac{D}{2} .\end{cases}
\end{aligned}
$$

Here the coefficients $\mathcal{A}^{\prime}=\mathcal{D}^{\prime}=0$ because of the asymptotic behavior that the field must vanish at $z= \pm \infty$. The other four coefficients are determined by the boundary conditions at the two interfaces $z= \pm D / 2$, where the field and its first derivative must fulfill the continuity conditions

$$
\begin{gathered}
\frac{2 \pi}{\varepsilon_{1} q} e^{q\left(D / 2+z^{\prime}\right)}+\mathcal{A} e^{-q D / 2}=\mathcal{B} e^{-q D / 2}+\mathcal{C} e^{q D / 2} \\
-2 \pi e^{q\left(D / 2+z^{\prime}\right)}+\varepsilon_{1} q \mathcal{A} e^{-q D / 2}=\varepsilon_{2} q \mathcal{B} e^{-q D / 2}-\varepsilon_{2} q \mathcal{C} e^{q D / 2} \\
\mathcal{B} e^{q D / 2}+\mathcal{C} e^{-q D / 2}=\mathcal{D} e^{-q D / 2} \\
\varepsilon_{2} q \mathcal{B} e^{q D / 2}-\varepsilon_{2} q \mathcal{C} e^{-q D / 2}=-q \mathcal{D} e^{-q D / 2}
\end{gathered}
$$

Then the coefficients read

$$
\begin{gathered}
\mathcal{A}=\frac{2 \pi}{\varepsilon_{1} q} \gamma\left[\frac{\varepsilon_{1}-\varepsilon_{2}}{\varepsilon_{1}+\varepsilon_{2}} e^{q\left(z^{\prime}+D\right)}+\frac{\varepsilon_{2}-1}{\varepsilon_{2}+1} e^{q\left(z^{\prime}-D\right)}\right], \\
\mathcal{B}=\frac{2 \pi}{q} \frac{2 \gamma}{\varepsilon_{1}+\varepsilon_{2}} \frac{\varepsilon_{2}-1}{\varepsilon_{2}+1} e^{q\left(z^{\prime}-D\right)}, \\
\mathcal{C}=\frac{2 \pi}{q} \frac{2 \gamma}{\varepsilon_{1}+\varepsilon_{2}} e^{q z^{\prime}}, \\
\mathcal{D}=\frac{2 \varepsilon_{2}}{\varepsilon_{1}+\varepsilon_{2}} \frac{2 \gamma}{\varepsilon_{2}+1} \frac{2 \pi}{q} e^{q z^{\prime}},
\end{gathered}
$$

where the variable $\gamma$ is defined in Eq. (5).

Performing the same procedure for $-D / 2<z^{\prime}<D / 2$ and $z^{\prime}>D / 2$, we can finally obtain the general form of the Coulomb kernel,

$$
K_{\mathrm{C}}\left(z, z^{\prime}, q, \omega\right)=\left\{\begin{array}{lc}
\frac{2 \pi}{\varepsilon_{1} q}\left[e^{-q\left|z-z^{\prime}\right|}+\gamma \frac{\varepsilon_{1}-\varepsilon_{2}}{\varepsilon_{1}+\varepsilon_{2}} e^{q\left(z+z^{\prime}+D\right)}+\gamma \frac{\varepsilon_{2}-1}{\varepsilon_{2}+1} e^{q\left(z+z^{\prime}-D\right)}\right], & z, z^{\prime}<-\frac{D}{2}, \\
\frac{2 \gamma}{\varepsilon_{1}+\varepsilon_{2}} \frac{2 \pi}{q}\left[e^{-q\left(z-z^{\prime}\right)}+\frac{\varepsilon_{2}-1}{\varepsilon_{2}+1} e^{q\left(z+z^{\prime}-D\right)}\right], & -\frac{D}{2}<z<\frac{D}{2}, z^{\prime}<-\frac{D}{2}, \\
\frac{2 \varepsilon_{2}}{\varepsilon_{1}+\varepsilon_{2}} \frac{2 \gamma}{\varepsilon_{2}+1} \frac{2 \pi}{q} e^{-q\left(z-z^{\prime}\right)}, & z>\frac{D}{2}, z^{\prime}<-\frac{D}{2}, \\
\frac{2 \gamma}{\varepsilon_{1}+\varepsilon_{2}} \frac{2 \pi}{q}\left[e^{q\left(z-z^{\prime}\right)}+\frac{\varepsilon_{2}-1}{\varepsilon_{2}+1} e^{q\left(z+z^{\prime}-D\right)}\right], & z<-\frac{D}{2},-\frac{D}{2}<z^{\prime}<\frac{D}{2}, \\
\frac{2 \pi}{\varepsilon_{2} q}\left\{e^{-q\left|z-z^{\prime}\right|}+\gamma \frac{\varepsilon_{2}-1}{\varepsilon_{2}+1}\left[e^{q\left(z+z^{\prime}-D\right)}+\frac{\varepsilon_{2}-\varepsilon_{1}}{\varepsilon_{2}+\varepsilon_{1}} e^{q\left(z-z^{\prime}-2 D\right)}\right]\right. & z>\frac{D}{2},-\frac{D}{2}<z^{\prime}<\frac{D}{2}, \\
\left.\quad+\gamma \frac{\varepsilon_{2}-\varepsilon_{1}}{\varepsilon_{2}+\varepsilon_{1}}\left[e^{-q\left(z+z^{\prime}+D\right)}+\frac{\varepsilon_{2}-1}{\varepsilon_{2}+1} e^{-q\left(z-z^{\prime}+2 D\right)}\right]\right\}, & z<-\frac{D}{2}, z^{\prime}>\frac{D}{2}, \\
\frac{2 \gamma}{\varepsilon_{2}+1} \frac{2 \pi}{q}\left[e^{-q\left(z-z^{\prime}\right)}+\frac{\varepsilon_{2}-\varepsilon_{1}}{\varepsilon_{2}+\varepsilon_{1}} e^{-q\left(z+z^{\prime}+D\right)}\right], & -\frac{D}{2}<z<\frac{D}{2}, z^{\prime}>\frac{D}{2}, \\
\frac{2 \varepsilon_{2}}{\varepsilon_{2}+1} \frac{2 \gamma}{\varepsilon_{1}+\varepsilon_{2}} \frac{2 \pi}{q} e^{q\left(z-z^{\prime}\right)}, & z, z^{\prime}>\frac{D}{2} . \\
\frac{2 \gamma}{\varepsilon_{2}+1} \frac{2 \pi}{q}\left[e^{q\left(z-z^{\prime}\right)}+\frac{\varepsilon_{2}-\varepsilon_{1}}{\varepsilon_{2}+\varepsilon_{1}} e^{-q\left(z+z^{\prime}+D\right)}\right], & \frac{D}{2}, \\
\frac{2 \pi}{q}\left[e^{-q\left|z-z^{\prime}\right|}+\gamma \frac{1-\varepsilon_{2}}{1+\varepsilon_{2}} e^{-q\left(z+z^{\prime}-D\right)}+\gamma \frac{\varepsilon_{2}-\varepsilon_{1}}{\varepsilon_{2}+\varepsilon_{1}} e^{-q\left(z+z^{\prime}+D\right)}\right], &
\end{array}\right.
$$

In contrast to the case of a free-standing jellium slab, the Coulomb kernel in the presence of dielectrics depends on the frequency $\omega$ (through the frequency-dependent dielectric functions) and the thickness of the film $D$.

*shiwu.gao@physics.gu.se

${ }^{1}$ Y. H. Yu, Y. Jiang, Z. Tang, Q. L. Guo, J. F. Jia, Q. K. Xue, K. H. Wu, and E. G. Wang, Phys. Rev. B 72, 205405 (2005).

${ }^{2}$ Y. H. Yu, Z. Tang, Y. Jiang, K. H. Wu, and E. G. Wang, Surf. Sci. 600, 4966 (2006).
${ }^{3}$ Y. Jiang, Y. H. Kim, S. B. Zhang, P. Ebert, S. Y. Yang, Z. Tang, K. H. Wu, and E. G. Wang, Appl. Phys. Lett. 91, 181902 (2007).

${ }^{4}$ Kehui $\mathrm{Wu}$ (private communication).

${ }^{5}$ H. J. Qin, Y. Gao, J. Teng, H. X. Xu, K. H. Wu, and S. W. Gao, Nano Lett. 10, 2961 (2010) 
${ }^{6}$ A. Politano, G. Chiarello, V. Formoso, R. G. Agostino, and E. Colavita, Phys. Rev. B 74, 081401 (2006).

${ }^{7}$ A. Politano, V. Formoso, and G. Chiarello, Plasmonics 3, 165 (2008).

${ }^{8}$ A. Politano, V. Formoso, E. Colavita, and G. Chiarello, Phys. Rev. B 79, 045426 (2009).

${ }^{9}$ A. Politano, V. Formoso, and G. Chiarello, J. Electron Spectrosc. Relat. Phenom. 173, 12 (2009).

${ }^{10}$ M. Rocca, Surf. Sci. Rep. 22, 1 (1995), and references therein.

${ }^{11}$ Z. Yuan and S. W. Gao, Phys. Rev. B 73, 155411 (2006).

${ }^{12}$ Z. Yuan and S. W. Gao, Surf. Sci. 602, 460 (2008).

${ }^{13}$ J. W. Gadzuk, Phys. Rev. B 1, 1267 (1970).

${ }^{14}$ A. G. Eguiluz, Phys. Rev. B 19, 1689 (1979); Phys. Rev. Lett. 51, 1907 (1983).

${ }^{15}$ F. Le, N. Z. Lwin, N. J. Halas, and P. Nordlander, Phys. Rev. B 76, 165410 (2007).

${ }^{16}$ A. G. Eguiluz and D. A. Campbell, Phys. Rev. B 31, 7572 (1985).

${ }^{17}$ A. G. Eguiluz, Phys. Rev. B 31, 3303 (1985).

${ }^{18}$ J. A. Gaspar, A. G. Eguiluz, K.-D. Tsuei, and E. W. Plummer, Phys. Rev. Lett. 67, 2854 (1991).

${ }^{19}$ A. Liebsch, Phys. Rev. Lett. 67, 2858 (1991).

${ }^{20}$ H. Ishida and A. Liebsch, Phys. Rev. B 45, 6171 (1992).

${ }^{21}$ H. Ishida and A. Liebsch, Phys. Rev. B 57, 12550 (1998).

${ }^{22}$ H. Ishida and A. Liebsch, Phys. Rev. B 57, 12558 (1998).

${ }^{23}$ V. M. Silkin, E. V. Chulkov, and P. M. Echenique, Phys. Rev. Lett. 93, 176801 (2004).

${ }^{24}$ S. W. Gao and D. S. Wang, Phys. Rev. B 44, 8812 (1991).

${ }^{25}$ L. P. Zhong, S. W. Gao, and D. S. Wang, Phys. Rev. B 47, 9791 (1993).

${ }^{26}$ T. Nagao, T. Hildebrandt, M. Henzler, and S. Hasegawa, Phys. Rev. Lett. 86, 5747 (2001).
${ }^{27}$ V. Zielasek, N. Rönitz, M. Henzler, and H. Pfnür, Phys. Rev. Lett. 96, 196801 (2006).

${ }^{28}$ Y. Liu, R. F. Willis, K. V. Emtsev, and Th. Seyller, Phys. Rev. B 78, 201403(R) (2008).

${ }^{29}$ Z. Y. Zhang, Q. Niu, and C.-K. Shih, Phys. Rev. Lett. 80, 5381 (1998).

${ }^{30}$ Z. Yuan and S. W. Gao, Comput. Phys. Commun. 180, 466 (2009).

${ }^{31}$ E. Prodan, A. Lee, and P. Nordlander, Chem. Phys. Lett. 360, 325 (2002).

${ }^{32}$ Ll. Serra, F. Garcías, J. Navarro, N. Barberán, M. Barranco, and M. Pi, Phys. Rev. B 46, 9369 (1992).

${ }^{33}$ J. Yan and S. W. Gao, Phys. Rev. B 78, 235413 (2008).

${ }^{34}$ A. Liebsch, Phys. Rev. B 48, 11317 (1993).

${ }^{35}$ P. J. Feibelman, Prog. Surf. Sci. 12, 287 (1982).

${ }^{36}$ A. Liebsch, Phys. Scr. 35, 354 (1987).

${ }^{37}$ A. Liebsch, Electronic Excitations at Metal Surfaces (Plenum, New York, 1997).

${ }^{38}$ D. L. Mills, Surf. Sci. 48, 59 (1975)

${ }^{39}$ E. D. Palik, Handbook of Optical Constants (Academic, London, 1985).

${ }^{40}$ B. Diaconescu, K. Pohl, L. Vattuone, L. Savio, P. Hofmann, V. M. Silkin, J. M. Pitarke, E. V. Chulkov, P. M. Echenique, D. Farias, and M. Rocca, Nature (London) 448, 57 (2007).

${ }^{41}$ S. J. Park and R. E. Palmer, Phys. Rev. Lett. 105, 016801 (2010).

${ }^{42}$ J. M. Pitarke, V. U. Nazarov, V. M. Silkin, E. V. Chulkov, E. Zaremba, and P. M. Echenique, Phys. Rev. B 70, 205403 (2004).

${ }^{43}$ V. M. Silkin, J. M. Pitarke, E. V. Chulkov, and P. M. Echenique, Phys. Rev. B 72, 115435 (2005).

${ }^{44}$ H. Wang, D. W. Brandl, P. Nordlander, and N. J. Halas, Acc. Chem. Res. 40, 53 (2007).

${ }^{45}$ A. Politano, V. Formoso, and G. Chiarello, Appl. Surf. Sci. 255, 6038 (2009). 